\title{
Veränderungen auf vielen Ebenen
}

\section{Peter Buess-Siegrist}

Dr. med., Facharzt für Psychiatrie und Psychotherapie, FMH

\author{
Menschen mit Hirnschädigungen haben mit ganz unterschiedlichen Beeinträchti- \\ gungen ihrer neuropsychologischen Funktionen zu kämpfen. Dadurch verändern \\ sich ihre Bedürfnisse. Für Angehörige ist es oft schwierig, den hirnverletzten Men- \\ schen zu verstehen. Aufklärungsarbeit soll hier Abhilfe schaffen: Der Verein Denk- \\ werk-Hirnverletzung Basel hat Erfahrungsberichte Betroffener herausgegeben.
}

Das Gehirn des Menschen kann in vielfacher Weise geschädigt werden. Gewalteinwirkung (Unfälle), Herzleiden mit Sauerstoffmangel (hypoxische Hirnschädigung), Tumorerkrankungen des ZNS, entzündliche Systemerkrankungen mit Hirnbefall, intrakranielle Blutungen sind die häufigsten Formen und zeigen eine riesige Bandbreite bezüglich Ätiologie und Pathogenese. Alle möglichen Ursachen können eine bleibende Hirnschädigung zur Folge haben. Nicht nur die Ursache spielt bei der Beurteilung von Hirnschädigungen eine entscheidende Rolle, sondern auch die Lokalisation der Schädigung (Hirnstamm, Frontalhirn, Temporallappen, tiefe Strukturen etc.).

Die Folgen einer Hirnschädigung lassen sich nicht kategorisieren. Häufige Beeinträchtigungen bei Hirnschädigungen sind: rasche Ermüdbarkeit, Konzentrationsschwierigkeiten, Aufmerksamkeitsprobleme, Orientierungsschwierigkeiten, Wortfindungsstörungen, Gedächtnisbeeinträchtigungen, Durchlässigkeit. Oft kommt es nach einer Hirnschädigung zur Entwicklung einer depressiven Störung. Auch die depressive Störung nach Hirnschädigung ist ein schwer fassbares Phänomen, wahrscheinlich liegt oft eine Kombination einer reaktiven (psychogenen) Depression mit einer hirnorganisch bedingten Entwicklung depressiver Symptome vor. Tatsache ist, dass sich eine depressive Störung bei Hirnschädigung eher als therapieresistent erweist. Das bedeutet, dass gerade bei Hirnschädigung eine antidepressive Kombinationsbehandlung nötig sein kann - ein unangenehmer Entscheid, da bei bereits geschädigtem Hirn der Einsatz von Psychopharmaka eine zusätzliche Belastung darstellt.

\section{Unsichtbare Schädigungen}

Als wäre es damit nicht genug, wird das Leben einer Person nach einer Hirnschädigung durch die Tatsache erschwert, dass oft wenig oder nichts von der Schädigung zu sehen ist. Die intellektuelle Leistungsfähigkeit muss nicht beeinträchtigt sein, sehr oft sind aber die Reizverarbeitung und die Fähigkeit zum Filtern (erhöhte Durchlässigkeit) derart verändert, dass eine quälende und anhaltende Erschöpfung respektive Ermüdung die Folge ist. Die uneingeschränkte intellektuelle Leistung kann zum Fehlschluss verleiten, es sei möglich, uneingeschränkt weiter zu arbeiten, beispielsweise in einer intellektuell anspruchsvollen Aufgabe. Das Umfeld kann nicht nachvollziehen, weshalb die Rückkehr in die Erwerbstätigkeit trotzdem nicht möglich sein soll. Wie aber soll die frühere Arbeitsbelastung bewältigt werden können, wenn zwar die intellektuelle Leistungsfähigkeit

\section{Zum Buch ... wieder leben lernen}

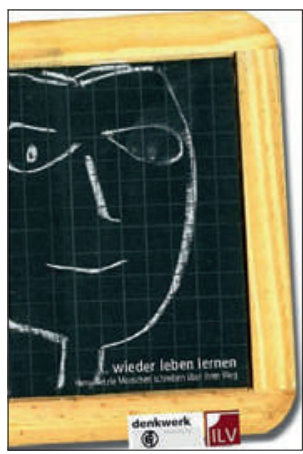

Verein Denkwerk-Hirnverletzung (Hrsg.)

.... wieder leben lernen

Hirnverletzte Menschen schreiben über ihren Weg. Basel: IL-Verlag; 2013. 142 Seiten. 25 CHF. ISBN 978-3-905955-80-4

In der Schweiz leben etwa 100000 Menschen mit einer Hirn verletzung. Jedes Jahr erleiden 3000 Menschen neu eine Hirnverletzung durch Unfälle, und 15000 werden betroffen durch Schlaganfälle, Hirnblutungen oder andere Ereignisse. Einschränkungen, die mit Verletzungen des Gehirns zusammenhängen, sind von aussen oft nicht sichtbar. Dies macht sie für Mitmenschen Betroffener schwer nachvollziehbar. Hirnverletzungen wirken sich auf alle Facetten des Menschseins aus. Menschen mit Hirnschädigungen müssen lernen, sich im Alltag wieder zurechtzufinden. Der Blick auf ihre Vergangenheit kann sich wandeln, aber auch die Zukunft hält Herausforderungen bereit, die nun anders bewältigt werden müssen. Um die Öffentlichkeit über das Leben von Menschen mit Hirnschädigungen zu informieren, hat der Verein Denkwerk-Hirnverletzung Basel das Buch ... wieder leben lernen herausgegeben. Betroffene berichten hier von ihren Erfahrungen. 
unbeeinträchtigt ist, aber rasche Ermüdung und eine ausgeprägte Antriebsstörung kombiniert mit Wortfindungsstörungen und verstärkter Vergesslichkeit kein kontinuierliches "Dranbleiben an einer Aufgabe» mehr erlauben? In diesem Sinne kommt es bei hirngeschädigten Menschen im Rahmen von Abklärungen und Begutachtungen oft zu Fehlschlüssen, welche das bereits tiefe Leiden betroffener Menschen unnötig verschärfen.

\section{Angehörige leiden mit}

Auch das soziale Umfeld wird durch eine Hirnschädigung erheblich in Mitleidenschaft gezogen. Für eine Partnerschaft ist die veränderte Persönlichkeit (als «Wesensänderung» treffend bezeichnet) eine extrem hohe Belastung. Eine Ehefrau meinte gut drei Jahre nach dem Erwachen ihres Mannes aus einem zweiwöchigen Koma nach Verkehrsunfall, jetzt (erst) sei ihr Mann zurückgekommen. Er war zwei Jahre zuvor bereits teilzeitlich erfolgreich an seine Arbeit zurückgekehrt. Aber die Situation blieb für seine Ehefrau unerträglich. Erst drei Jahre nach dem Unfall bekam die Partnerin den Eindruck, ihren ursprünglichen Partner zumindest teilweise wieder zurückerhalten zu haben. Obwohl die Belastung für Angehörige unermesslich sein kann, wird dies selten berücksichtigt. So stellte die Ehefrau eines Mannes mit schwerer Hirnschädigung fest, dass sie 10 Jahre nach dem Unfall ihres Mannes zum ersten Mal die Gelegenheit hatte, ein Gespräch über die Folgen der Hirnschädigung ihres Mannes zu führen. Und zwar im Rahmen einer Begutachtung, bei der sie zur Erhebung der Fremdauskunft mit einbezogen wurde.

\section{Wie können Ärzte helfen?}

Wie erkennt ein Hausarzt, dass bei einer Person Folgen einer Hirnschädigung vorliegen? Wie soll mit konflikthaften Situationen (Beziehungskonflikte, aggressives Verhalten) umgegangen werden? Wenn Zielsetzungen besprochen werden, wird sich immer wieder zeigen, dass wenig davon erreicht werden kann - wie soll dies akzeptiert, verstanden werden? In aller Regel setzt sich ein Mensch mit den Folgen einer Hirnschädigung schon in der Rehabilitationsphase selber massiv unter Druck, wieder die alte Person sein zu müssen. Das heisst, die betroffene Person baut eine immense Abwehr gegen die irreversiblen Folgen der Hirnschädigung auf. Diese psychische Reaktion ist nicht sichtbar, schon gar nicht in bildgebenden Verfahren. Diese Form psychischer Abwehr wird sogar oft missverstanden als bewundernswerte Willensanstrengung der betroffenen Person, alle
Energie für eine erfolgreiche Rehabilitation einzusetzen. Daraus wird quasi ein vorbildlicher Patient - hinter der Kulisse bauen sich Ängste, Verzweiflung, Hilflosigkeit auf. Denn diese Dynamik ist oft gar nicht so verständlich, wie hier beschrieben, sondern entwickelt sich in einem diffusen Nebel - denn die Kognition bleibt ja beeinträchtigt und deshalb ist es gar nicht möglich, dieses Auseinanderklaffen von Wollen und Können bewusst wahrzunehmen und zu verstehen. Der Prozess der Abwehr kann sich über Monate, ja Jahre hinziehen und wird schliesslich gekrönt von einem depressiven $\mathrm{Zu}$ sammenbruch, der für das Umfeld einfach nur unverständlich ist - nachdem doch alles so gut lief. Solche Prozesse erzeugen über lange Zeiträume ein unerträgliches Leiden.

Der Kern des Leidens liegt im «Unsichtbaren» und im "Unsagbaren». Deshalb ist oft eine sehr einfach wirkende und doch sehr potent wirksame Intervention der Hinweis aus der ärztlichen Ecke: «Sie leiden an einer Hirnschädigung, deshalb ...» Verständlicherweise wird lieber geschont vor der herben Wahrheit, aber diese Formulierung kann zutiefst klärend und hilfreich sein, denn sie vertreibt den Nebel von Abwehr und beeinträchtigter Kognition. Es ist also wichtig, ausgehend vom Befund einer neuropsychologischen $\mathrm{Ab}$ klärung den Zusammenhang aufzuzeigen zwischen Hirnschädigung und der Reizverarbeitung, Durchlässigkeit, den Störungen im Antrieb, im Gedächtnis, in der Konzentration, in der geteilten Aufmerksamkeit usw. Das Benennen der Einschränkungen ist für die betroffene Person gar nicht möglich, sie erlebt nur die Folgen, ohne jedoch verstehen zu können, was Sache ist. Die Begriffe «Hirnschädigung», «Durchlässigkeit», «Reizverarbeitung», "Aufmerksamkeitsbreite» gehören in das existentielle Vokabular, sich selber verstehen zu können.

Ein Letztes: Wir leben mit einer Art innerer Heimat. Wir sind in Erinnerungen, in Wertsystemen verortet. Beschrieben wird dies auch als narrativer Schwerpunkt [1]. Mit dem Erzählen entwickeln wir unsere Identität. Wenn die Identität infolge einer Hirnschädigung verloren gegangen ist, wird das Erzählen existentiell wichtig. Deshalb ist die Psychotherapie über einen längeren Zeitraum nach einer Hirnschädigung unverzichtbar. Natürlich helfen viele andere Bezüge, die innere Heimat wieder aufzubauen - die Psychotherapie ist nur ein Teil von einem jahrelangen Prozess.

Und zuallerletzt: Mit Geduld zuhören, auf Details achten, ist für die Ärztin wie auch die betroffene Person äusserst hilfreich. Denn die betroffene Person ist ExpertIn in eigener Sache. 